\title{
Approximate Cauchy functional inequality in quasi-Banach spaces
}

Hark-Mahn Kim and Eunyoung Son ${ }^{*}$

* Correspondence:

sey8405@hanmail.net

Department of Mathematics,

Chungnam National University, 79

Daehangno, Yuseong-gu, Daejeon

305-764, Korea

\section{Abstract}

In this article, we prove the generalized Hyers-Ulam stability of the following Cauchy functional inequality:

$$
\|f(x)+f(y)+n f(z)\| \leq n f\left\|\left(\frac{x+y}{n}+x\right)\right\|
$$

in the class of mappings from $n$-divisible abelian groups to $p$-Banach spaces for any fixed positive integer $n \geq 2$.

\section{Introduction}

The stability problem of functional equations originated from a question of Ulam [1] concerning the stability of group homomorphisms.

We are given a group $G_{1}$ and a metric group $G_{2}$ with metric $\rho(\cdot, \cdot)$. Given $\epsilon>0$, does there exist a $\delta>0$ such that if $f: G_{1} \rightarrow G_{2}$ satisfies $\rho(f(x y), f(x) f(y))<\delta$ for all $x, y \in G_{1}$, then a homomorphism $h: G_{1} \rightarrow G_{2}$ exists with $\rho(f(x), h(x))<\epsilon$ for all $x \in G_{1}$ ?

In other words, we are looking for situations when the homomorphisms are stable, $\mathrm{i}$. e., if a mapping is almost a homomorphism, then there exists a true homomorphism near it.

In 1941, Hyers [2] considered the case of approximately additive mappings between Banach spaces and proved the following result. Suppose that $E_{1}$ and $E_{2}$ are Banach spaces and $f: E_{1} \rightarrow E_{2}$ satisfies the following condition: there is a constant $\epsilon \geq 0$ such that

$$
|f(x+y)-f(x)-(y)| \mid \leq \varepsilon
$$

for all $x, y \in E_{1}$. Then, the limit $h(x)=\lim _{n \rightarrow \infty} \frac{f\left(2^{n} x\right)}{2^{n}}$ exists for all $x \in E_{1}$, and it is a unique additive mapping $h: E_{1} \rightarrow E_{2}$ such that $\|f(x)-h(x)\| \leq \epsilon$.

The method which was provided by Hyers, and which produces the additive mapping $h$, was called a direct method. This method is the most important and most powerful tool for studying the stability of various functional equations. Hyers' theorem was generalized by Aoki [3] and Bourgin [4] for additive mappings by considering an unbounded Cauchy difference. In 1978, Rassias [5] also provided a generalization of Hyers' theorem for linear mappings which allows the Cauchy difference to be unbounded like this $\|x\|^{p}+\|y\|^{p}$. Let $E_{1}$ and $E_{2}$ be two Banach spaces and $f: E_{1} \rightarrow$ $E_{2}$ be a mapping such that $f(t x)$ is continuous in $t \in \mathbf{R}$ for each fixed $x$. Assume that

(c) 2011 Kim and Son; licensee Springer. This is an Open Access article distributed under the terms of the Creative Commons Attribution License (http://creativecommons.org/licenses/by/2.0), which permits unrestricted use, distribution, and reproduction in any medium, provided the original work is properly cited. 
there exists $\epsilon>0$ and $0 \leq p<1$ such that

$$
\|f(x+y)-f(x)-f(y)\| \leq \varepsilon\left(\|x\|^{p}+\|y\|^{p}\right), \quad \forall x, y \in E_{1} .
$$

Then, there exists a unique $\mathbf{R}$-linear mapping $T: E_{1} \rightarrow E_{2}$ such that

$$
\|f(x)-T(x)\| \leq \frac{2}{2-2^{p}}\|x\|^{p}
$$

for all $x \in E_{1}$. A generalized result of Rassias' theorem was obtained by Găvruta in [6] and Jung in [7]. In 1990, Rassias [8] during the 27th International Symposium on Functional Equations asked the question whether such a theorem can also be proved for $p \geq 1$. In 1991, Gajda [9], following the same approach as in [5], gave an affirmative solution to this question for $p>1$. It was shown by Gajda [9], as well as by Rassias and [001] emrl [10], that one cannot prove a Rassias' type theorem when $p=1$. The counterexamples of Gajda [9], as well as of Rassias and [001]emrl [10], have stimulated several mathematicians to invent new approximately additive or approximately linear mappings. In particular, Rassias $[11,12]$ proved a similar stability theorem in which he replaced the unbounded Cauchy difference by this factor $\left.\|x\|\right|^{p}|| y \|^{q}$ for $p, q \in \mathbf{R}$ with $p$ $+q \neq 1$.

Let $G$ be an $n$-divisible abelian group $n \in \mathbf{N}$ (i.e., $a \mapsto n a: G \rightarrow G$ is a surjection ) and $X$ be a normed space with norm $\|\cdot\|$. Now, for a mapping $f: G \rightarrow X$, we consider the following generalized Cauchy-Jensen equation

$$
f(x)+f(y)+n f(z)=n f\left(\frac{x+y}{n}+z\right), \quad n \geq 2
$$

for all $x, y, z \in G$, which has been introduced in [13].

Proposition 1.1. For a mapping $f: G \rightarrow X$, the following statements are equivalent.
(a) $f$ is additive,
(b) $f(x)+f(y)+n f(z)=n f\left(\frac{x+y}{n}+z\right)$,
(c) $\|f(x)+f(y)+n f(z)\| \leq\left\|n f\left(\frac{x+y}{n}+z\right)\right\|$

for all $x, y, z \in G$.

As a special case for $n=2$, the generalized Hyers-Ulam stability of functional equation (b) and functional inequality (c) has been presented in [13]. We remark that there are some interesting papers concerning the stability of functional inequalities and the stability of functional equations in quasi-Banach spaces [14-18]. In this article, we are going to improve the theorems given in [13] without using the oddness of approximate additive functions concerning the functional inequality (c) for a more general case.

\section{Generalized Hyers-Ulam stability of (c)}

We recall some basic facts concerning quasi-Banach spaces and some preliminary results. Let $X$ be a real linear space. A quasi-norm is a real-valued function on $X$ satisfying the following: 
(1) $\|x\| \geq 0$ for all $x \in X$ and $\|x\|=0$ if and only if $x=0$.

(2) $\| \lambda x||=|\lambda||| x||$ for all $\lambda \in \mathbf{R}$ and all $x \in X$.

(3) There is a constant $M \geq 1$ such that $\|x+y\| \leq M(\|x\|+\|y\|)$ for all $x, y \in X$.

The pair $(X,\|\cdot\|)$ is called a quasi-normed space if $\|\cdot\|$ is a quasi-norm on $X$ $[19,20]$. The smallest possible $M$ is called the modulus of concavity of $\|\cdot\|$. A quasiBanach space is a complete quasi-normed space.

A quasi-norm $\|\cdot\|$ is called a $p$-norm $(0<p \leq 1)$ if

$$
\|x+y\|^{p} \leq\left.\|x\|\right|^{p}+\|y\|^{p}
$$

for all $x, y \in X$. In this case, a quasi-Banach space is called a $p$-Banach space.

Given a $p$-norm, the formula $d(x, y):=\|x-y\|^{p}$ gives us a translation invariant metric on $X$. By the Aoki-Rolewicz theorem [20], each quasi-norm is equivalent to some $p$-norm (see also [19]). Since it is much easier to work with $p$-norms, henceforth, we restrict our attention mainly to $p$-norms. We observe that if $x_{1}, x_{2}, \ldots, x_{n}$ are nonnegative real numbers, then

$$
\left(\sum_{i=1}^{n} x_{i}\right)^{p} \leq \sum_{i=1}^{n} x_{i}^{p},
$$

where $0<p \leq 1[21]$.

From now on, let $G$ be an $n$-divisible abelian group for some positive integer $n \geq 2$, and let $Y$ be a $p$-Banach space with the modulus of concavity $M$.

Theorem 2.1. Suppose that a mapping $f: G \rightarrow Y$ with $f(0)=0$ satisfies the functional inequality

$$
\|f(x)+f(y)+n f(z)\| \leq\left\|n f\left(\frac{x+y}{n}+z\right)\right\|+\varphi(x, y, z)
$$

for all $x, y, z \in G$, and the perturbing function $\phi: G^{3} \rightarrow \mathrm{R}^{+}$satisfies

$$
\Phi(x, y, z):=\sum_{i=0}^{\infty} \frac{\varphi\left(n^{i} x, n^{i} y, n^{i} z\right)^{p}}{n^{i p}}<\infty
$$

for all $x, y, z \in G$. Then, there exists a unique additive mapping $h: G \rightarrow Y$, defined as $h(x)=\lim _{k \rightarrow \infty} \frac{f\left(n^{k} x\right)-f\left(-n^{k} x\right)}{2 n^{k}}$, such that

$$
\|f(x)-h(x)\| \leq \frac{M^{2}}{2 n}[\Phi(n x, 0,-x)+\Phi(-n x, 0, x)]^{\frac{1}{p}}+\frac{M}{2} \varphi(x,-x, 0)
$$

for all $x \in G$.

Proof. Let $y=-x, z=0$ in (1) and dividing both sides by 2 , we have

$$
\left\|\frac{f(x)+f(-x)}{2}\right\| \leq \frac{\varphi(x,-x, 0)}{2}
$$

for all $x \in G$. Replacing $x$ by $n x$ and letting $y=0$ and $z=-x$ in (1), we get

$$
\|f(n x)+n f(-x)\| \leq \varphi(n x, 0,-x)
$$


for all $x \in G$. Replacing $x$ by $-x$ in (4), one has

$$
\|f(-n x)+n f(x)\| \leq \varphi(-n x, 0, x)
$$

for all $x \in$ G. Put $g(x)=\frac{f(x)-f(-x)}{2}$. Combining (4) and (5) yields

$$
\|n g(x)-g(n x)\| \leq \frac{M}{2}(\varphi(n x, 0,-x)+\varphi(-n x, 0, x))
$$

that is,

$$
\left\|g(x)-\frac{1}{n} g(n x)\right\| \leq \frac{M}{2 n}(\varphi(n x, 0,-x)+\varphi(-n x, 0, x))
$$

for all $x \in G$. It follows from (6) that

$$
\begin{aligned}
& \left\|\frac{g\left(n^{l} x\right.}{n^{l}}-\frac{g\left(n^{m} x\right)}{n^{m}}\right\|^{p} \\
\leq & \sum_{k=l}^{m-1}\left\|\frac{1}{n^{k}} g\left(n^{k} x\right)-\frac{1}{n^{k+1}} g\left(n^{k+1} x\right)\right\|^{p} \\
= & \sum_{k=1}^{m-1} \frac{1}{n^{k p}}\left\|g\left(n^{k} x\right)-\frac{1}{n} g\left(n^{k+1} x\right)\right\|^{p} \\
\leq & \sum_{k=1}^{m-1} \frac{M^{p}}{2^{p} n^{(k+1)_{p}}}\left[\varphi\left(n^{k+1} x, 0,-n^{k} x\right)^{p}+\varphi\left(-n^{k+1} x, 0, n^{k} x\right)^{p}\right]
\end{aligned}
$$

for all nonnegative integers $m$ and $l$ with $m>l \geq 0$ and $x \in G$. Since the right-hand side of (7) tends to zero as $l \rightarrow \infty$, we obtain the sequence $\left\{\frac{g\left(n^{m} x\right.}{n^{m}}\right\}$ is Cauchy for all $x$ $\in G$. Because of the fact that $Y$ is complete, it follows that the sequence $\left\{\frac{g\left(n^{m} x\right.}{n^{m}}\right\}$ converges in $Y$. Therefore, we can define a function $h: G \rightarrow Y$ by

$$
h(x)=\lim _{m \rightarrow \infty} \frac{g\left(n^{m} x\right)}{n^{m}}=\lim _{m \rightarrow \infty} \frac{f\left(n^{m} x\right)-f\left(-n^{m} x\right)}{2 n^{m}}, \quad x \in G .
$$

Moreover, letting $l=0$ and taking $m \rightarrow \infty$ in (7), we get

$$
\left\|\frac{f(x)-f(-x)}{2}-h(x)\right\| \leq\|g(x)-h(x)\| \leq \frac{M}{2 n}[\Phi(n x, 0-x)+\Phi(-n x, 0, x)]^{\frac{1}{p}}
$$

for all $x \in G$. It follows from (3) and (8) that

$$
\|f(x)-h(x)\| \leq \frac{M^{2}}{2 n}[\Phi(n x, 0,-x)+\Phi(-n x, 0, x)]^{\frac{1}{p}}+\frac{M}{2} \varphi(x,-x, 0)
$$

for all $x \in G$. 
It follows from (1) and (4) that

$$
\begin{aligned}
\|h(x)+h(y)-h(x+y)\|^{p}= & \|h(x)+h(y)+h(-x-y)\|^{p} \\
= & \lim _{k \rightarrow \infty} \frac{1}{n^{k p}}\left\|g\left(n^{k} x\right)+g\left(n^{k} y\right)+g\left(-n^{k}(x+y)\right)\right\|^{p} \\
\leq & \lim _{k \rightarrow \infty} \frac{1}{2^{p} n^{k p}}\left(\left\|f\left(n^{k} x\right)+f\left(n^{k} y\right)+n f\left(-n^{k-1}(x+y)\right)\right\|^{p}\right. \\
& +\left\|-f\left(-n^{k} x\right)-f\left(-n^{k} y\right)-n f\left(n^{k-1}(x+y)\right)\right\|^{p} \\
& +\left\|n f\left(n^{k-1}(x+y)\right)+f\left(-n^{k}(x+y)\right)\right\|^{p} \\
& +\left\|-n f\left(-n^{k-1}(x+y)\right)+f\left(n^{k}(x+y)\right)\right\|^{p} \\
\leq & \lim _{k \rightarrow \infty} \frac{1}{2^{p} n^{k p}}\left(\varphi\left(n^{k} x, n^{k} \gamma,-n^{k-1}(x+y)\right)^{p}+\varphi\left(-n^{k} x,-n^{k} y, n^{k-1}(x+y)\right)^{p}\right. \\
& \left.+\varphi\left(-n^{k}(x+y), 0, n^{k-1}(x+y)\right)^{p}+\varphi\left(n^{k}(x+y), 0,-n^{k-1}(x+y)\right)^{p}\right) \\
= & 0
\end{aligned}
$$

for all $x, y \in G$. This implies that the mapping $h$ is additive.

Next, let $h^{\prime}: G \rightarrow Y$ be another additive mapping satisfying

$$
\left\|f(x)-h^{\prime}(x)\right\| \leq \frac{M^{2}}{2 n}[\Phi(n x, 0,-x)+\Phi(-n x, 0, x)]^{\frac{1}{p}}+\frac{M}{2} \varphi(x,-x, 0)
$$

for all $x \in G$. Then, we have

$$
\begin{aligned}
\left\|h(x)-h^{\prime}(x)\right\|^{p} & =\left\|\frac{1}{n^{k}} h\left(n^{k} x\right)-\frac{1}{n^{k}} h^{\prime}\left(n^{k} x\right)\right\|^{p} \\
& \leq \frac{1}{n^{k p}}\left(\left\|h\left(n^{k} x\right)-f\left(n^{k} x\right)\right\|^{p}+\left\|f\left(n^{k} x\right)-h^{\prime}\left(n^{k} x\right)\right\|^{p}\right) \\
& \leq \frac{2 M^{2 p}}{2^{p} n^{(k+1)_{p}}}\left[\Phi\left(n^{k+1} x, 0,-n^{k} x\right)+\Phi\left(-n^{k+1} x, 0, n^{k} x\right)\right]+\frac{2 M^{p}}{2^{p} n^{k p}} \varphi\left(n^{k} x,-n^{k} x, 0\right)^{p} \\
& =\sum_{i=k}^{\infty} \frac{2 M^{2 p}}{2^{p} n^{(i+1) p}}\left[\varphi\left(n^{i+1} x, 0,-n^{i} x\right)^{p}+\varphi\left(-n^{i+1} x, 0, n^{i} x\right)^{p}\right]+\frac{2 M^{p} \varphi\left(n^{k} x,-n^{k} x, 0\right)^{p}}{2^{p} n^{k p}}
\end{aligned}
$$

for all $k \in \mathrm{N}$ and all $x \in G$. Taking the limit as $k \rightarrow \infty$, we conclude that

$$
h(x)=h^{\prime}(x)
$$

for all $x \in G$. This completes the proof.

Suppose that $X$ is a normed space in the following corollaries. If we put $\phi(x, y, z):=$ $\theta\left(\|x\|^{q}|| y||^{r}|| z \|^{s}\right)$ and $\phi(x, y, z):=\theta\left(\|x\|^{q}+\|y\|^{r}+\|z\|^{s}\right)$ in Theorem 2.1, respectively, then we get the following Corollaries 2.2 and 2.3.

Corollary 2.2. Let $q+r+s<1, q, r, s>0, \theta>0$. If a mapping $f: X \rightarrow Y$ with $f(0)=$ 0 satisfies the following functional inequality:

$$
\|f(x)+f(y)+n f(z)\| \leq\left\|n f\left(\frac{x+y}{n}+x\right)\right\|+\theta\left(\left\|x||^{q}|| y \mid\right\|^{r}\|z\|^{s}\right.
$$

for all $x, y, z \in X$, then $f$ is additive.

Corollary 2.3. Let $0<q, r, s<1, \theta_{1}, \theta_{2}>0$. If a mapping $f: X \rightarrow Y$ with $f(0)=0$ satisfies the following functional inequality:

$$
\|f(x)+f(y)+n f(z)\| \leq\left\|n f\left(\frac{x+y}{n}+z\right)\right\|+\theta_{1}\left(\|x\|^{q}+\|y\|^{r}+\|z\|^{s}\right)+\theta_{2}
$$

for all $x, y, z \in X$, then there exists a unique additive mapping $h: X \in Y$, defined as $h(x)=\lim _{k \rightarrow \infty} \frac{f\left(n^{k} x\right)-f\left(-n^{k} x\right)}{2 n^{k}}$, such that 


$$
\begin{aligned}
\|f(x)-h(x)\| \leq & \frac{M^{2} \sqrt[p]{2}}{2}\left(\frac{n^{p q} \theta_{1}^{p} \|\left. x\right|^{p q}}{n^{p}-n^{p q}}+\frac{\theta_{1}^{p} \|\left. x\right|^{p s}}{n^{p}-n^{p s}}+\frac{\theta_{2}^{p}}{n^{p}-1}\right)^{\frac{1}{p}} \\
& +\frac{M}{2}\left(\theta_{1}\|x\|^{q}+\theta_{1}\|x\|^{r}+\theta_{2}\right)
\end{aligned}
$$

for all $x \in X$.

Noting the inequality

$$
\|f(n x)-n f(x)\| \leq M[\varphi(n x, 0,-x)+n \varphi(x,-x, 0)]
$$

according to the inequalities (3) and (4), then we can similarly prove another stability theorem under the same condition as in Theorem 2.1:

Remark 2.4. Let $\phi: G^{3} \rightarrow \mathbf{R}+$ and $f: G \rightarrow Y$ satisfy the assumptions of Theorem 2.1. Then, there exists a unique additive mapping $h: \mathrm{G} \rightarrow Y$, defined by $h(x)=\lim _{k \rightarrow \infty} \frac{f\left(n^{k} x\right)}{n^{k}}$, such that

$$
\|f(x)-h(x)\| \leq \frac{M}{n}\left[\Phi(n x, 0,-x)+n^{p} \Phi(x,-x, 0)\right]^{\frac{1}{p}}
$$

for all $x \in G$ using the similar argument to Theorem 2.1.

In particular, if a mapping $f: X \rightarrow Y$ with $f(0)=0$ satisfies the following functional inequality:

$$
\|f(x)+f(y)+n f(z)\| \leq\left\|n f\left(\frac{x+y}{n}+z\right)\right\|+\theta_{1}\left(\|x\|^{q}+\|y\|^{r}+\|z\|^{s}\right)+\theta_{2}
$$

for all $x, y, z$ in a normed space $X$, where $0<q, r, s<1, \theta_{1}, \theta_{2}>0$, then there exists a unique additive mapping $h: X \rightarrow Y$ such that

$$
\|f(x)-h(x)\| \leq M\left(\frac{\left(n^{p q}+n^{p}\right) \theta_{1}^{p}\|x \mid\|^{p q}}{n^{p}-n^{p q}}+\frac{n^{p} \theta_{1}^{p}\|x\|^{p r}}{n^{p}-n^{p r}}+\frac{\theta_{1}^{p}\|x\|^{p s}}{n^{p}-n^{p s}}+\frac{\left(1+n^{p}\right) \theta_{2}^{2}}{n^{p}-1}\right)^{\frac{1}{p}}
$$

for all $x \in X$.

We may obtain more simple and sharp approximation than that of Theorem 2.1 for the stability result under the oddness condition.

Remark 2.5. Let $\phi: G^{3} \rightarrow \mathrm{R}^{+}$and $f: G \rightarrow Y$ satisfy the assumptions of Theorem 2.1. Moreover, if the mapping $f$ is odd, then there exists a unique additive mapping $h: G$ $\rightarrow Y$, defined by $h(x)=\lim _{k \rightarrow \infty} \frac{f\left(n^{k} x\right)}{n^{k}}$, such that

$$
\|f(x)-h(x)\| \leq \frac{1}{n} \Phi(n x, 0,-x)^{\frac{1}{p}}
$$

for all $x \in G$.

Now, we consider another stability result of functional inequality (c) in the followings. 
Theorem 2.6. Suppose that a mapping $f: G \rightarrow Y$ satisfies

$$
\|f(x)+f(y)+n f(z)\| \leq\left\|n f\left(\frac{x+y}{n}+z\right)\right\|+\varphi(x, y, z)
$$

and the perturbing function $\phi: G^{3} \rightarrow \mathrm{R}^{+}$is such that

$$
\Psi(x, y, z):=\sum_{i=1}^{\infty} n^{i p} \varphi\left(\frac{x}{n^{i}}, \frac{y}{n^{i}}, \frac{z}{n^{i}}\right)^{p}<\infty
$$

for all $x, y, z \in G$. Then, there exists a unique additive mapping $h: G \rightarrow Y$, defined $h(x) \lim _{k \rightarrow \infty} \frac{n^{k}}{2}\left(f\left(\frac{x}{n^{k}}\right)-f\left(-\frac{x}{n^{k}}\right)\right)$, such that

$$
\|f(x)-h(x)\| \leq \frac{M^{2}}{2 n}[\Psi(n x, 0,-x)+\Psi(-n x, 0, x)]^{\frac{1}{p}}+\frac{M}{2} \varphi(x,-x, 0)
$$

for all $x \in G$.

Proof. We observe that $f(0)=0$ because of $\phi(0,0,0)=0$ by the convergence of $\Psi(0,0,0)<\infty$. Now, combining (4) and (5) yields the functional inequality

$$
\left\|g(x)-n g\left(\frac{x}{n}\right)\right\| \leq \frac{M}{2}\left(\varphi\left(x, 0,-\frac{x}{n}\right)+\varphi\left(-x, 0, \frac{x}{n}\right)\right) .
$$

where $g(x)=\frac{f(x)-f(-x)}{2}, x \in G$. It follows from the last inequality that

$$
\left\|g(x)-n^{m} g\left(\frac{x}{n^{m}}\right)\right\|^{p} \leq \frac{M^{p}}{2^{p}} \sum_{i=0}^{m-1} n^{i p}\left[\varphi\left(\frac{x}{n^{i}}, 0,-\frac{x}{n^{i+1}}\right)^{p}+\varphi\left(-\frac{x}{n^{i}}, 0, \frac{x}{n^{i+1}}\right)^{p}\right]
$$

for all $x L G$.

The remaining proof is similar to the corresponding proof of Theorem 2.1. This completes the proof.

Suppose that $X$ is a normed space in the following corollaries. If we put $\phi(x, y, z):=$ $\theta\left(\|x\|^{q}|| y\left\|^{r}|| z\right\|^{s}\right)$ and $\phi(x, y, z):=\theta\left(\|x\|^{q}+\|y\|^{r}+\|z\|^{s}\right)$ in Theorem 2.6, respectively, then we get the following Corollaries 2.7 and 2.8 .

Corollary 2.7. Let $q+r+s>1, q, r, s>0, \theta>0$. If a mapping $f: X \rightarrow Y$ satisfies the following functional inequality:

$$
\|f(x)+f(y)+n f(z)\| \leq\left\|n f\left(\frac{x+y}{n}+x\right)\right\|+\theta\left(\left\|\left.x\right|^{q}|| y\right\|^{r}\|z\|^{s}\right.
$$

for all $x, y, z \in \mathrm{X}$, then $f$ is additive.

Corollary 2.8. Let $q, r, s>1, \theta_{1}>0$. If a mapping $f: X \rightarrow Y$ satisfies the following functional inequality:

$$
\|f(x)+f(y)+n f(z)\| \leq\left\|n f\left(\frac{x+y}{n}+z\right)\right\|+\theta_{1}\left(\|x\|^{q}+\|y\|^{r}+\|z\|^{s}\right)
$$

for all $x, y, z \in X$, then there exists a unique additive mapping $h: X \rightarrow Y$, defined as $h(x) \lim _{k \rightarrow \infty} \frac{n^{k}}{2}\left(f\left(\frac{x}{n^{k}}\right)-f\left(-\frac{x}{n^{k}}\right)\right)$, such that 


$$
\|f(x)-h(x)\| \leq \frac{M^{2} \sqrt[p]{2 \theta_{1}}}{2}\left(\frac{n^{p q}|| x \|^{p q}}{n^{p q}-n^{p}}+\frac{\|\left. x\right|^{p s}}{n^{p s}-n^{p}}\right)^{\frac{1}{p}}+\frac{M \theta_{1}}{2}\left(\|x\|^{q}+\|x\|^{r}\right)
$$

for all $x \in X$.

We can similarly prove another stability theorem under somewhat different conditions as follows:

Remark 2.9. Let $\phi: G^{3} \rightarrow \mathrm{R}^{+}$and $f: G \rightarrow Y$ satisfy the assumptions of Theorem 2.6. Then, there exists a unique additive mapping $h: G \rightarrow Y$, defined by $h(x)=$ $h(x)=\lim _{k \rightarrow \infty} n^{k} f\left(\frac{x}{n^{k}}\right)$, such that

$$
\|f(x)-h(x)\| \leq \frac{M}{n}\left[\Psi(n x, 0,-x)+n^{p} \Psi(x,-x, 0)\right]^{\frac{1}{p}}
$$

for all $x \in G$.

In particular, if a mapping $f: X \rightarrow Y$ satisfies the following functional inequality:

$$
\|f(x)+f(y)+n f(z)\| \leq\left\|n f\left(\frac{x+y}{n}+z\right)\right\|+\theta_{1}\left(\|x\|^{q}+\|y\|^{r}+\|z\|^{s}\right)
$$

for all $x, y, z$ in a normed space $X$, where $q, r, s>1, \theta_{1}>0$, then there exists a unique additive mapping $h: X \rightarrow Y$ such that

$$
\|f(x)-h(x)\| \leq M \theta_{1}\left(\frac{\left(n^{p q}+n^{p}\right)\|x\|^{p q}}{n^{p q}-n^{p}}+\frac{\|x\|^{p s}}{n^{p s}-n^{p}}+\frac{n^{p}|| x|| p r}{n^{p r}-n^{p}}\right)^{\frac{1}{p}}
$$

for all $x \in X$.

We may obtain more simple and sharp approximation than that of Theorem 2.6 for the stability result under the oddness condition.

Remark 2.10. Let $\phi: G^{3} \rightarrow \mathrm{R}^{+}$and $f: G \rightarrow Y$ satisfy the assumptions of Theorem 2.6. If the mapping $f$ is odd, then there exists a unique additive mapping $h: G \rightarrow Y$, defined by $h(x)=\lim _{k \rightarrow \infty} n^{k} f\left(\frac{x}{n^{k}}\right)$, such that

$$
\|f(x)-h(x)\| \leq \frac{1}{n} \Psi(n x, 0,-x)^{\frac{1}{p}}
$$

for all $x \in G$.

\section{Alternative generalized Hyers-Ulam stability of (c)}

From now on, we investigate the generalized Hyers-Ulam stability of the functional inequality (c).

Theorem 3.1. Suppose that a mapping $f: G \rightarrow Y$ with $f(0)=0$ satisfies the functional inequality

$$
\|f(x)+f(y)+n f(z)\| \leq\left\|n f\left(\frac{x+y}{n}+z\right)\right\|+\varphi(x, y, z)
$$

for all $x, y, z \in G$ and there exists a constant $L$ with $0<L<1$ for which the perturbing function $\phi: G^{3} \rightarrow \mathbf{R}^{+}$satisfies 


$$
\varphi(n x, n y, n z) \leq n L \varphi(x, y, z)
$$

for all $x, y, z \in G$. Then, there exists a unique additive mapping $h: G \rightarrow Y$, defined as $h(x)=\lim _{k \rightarrow \infty} \frac{f\left(n^{k} x\right)-f\left(-n^{k} x\right)}{2 n^{k}}$, such that

$$
\|f(x)-h(x)\| \leq \frac{M^{2}}{2 n \sqrt[p]{1-L^{p}}}[\varphi(n x, 0,-x)+\varphi(-n x, 0, x)]+\frac{M}{2} \varphi(x,-x, 0)
$$

for all $x \in G$.

Proof. It follows from (7) and (12) that

$$
\begin{aligned}
& \left\|\frac{g\left(n^{1} x\right)}{n^{1}}-\frac{g\left(n^{m} x\right)}{n^{m}}\right\|^{p} \\
\leq & \sum_{k=1}^{m-1} \frac{M^{p}}{2^{p} n^{(k+1) p}}\left[\varphi\left(n^{k+1} x, 0,-n^{k} x\right)+\varphi\left(-n^{k+1} x, 0, n^{k} x\right)\right]^{p} \\
\leq & \sum_{k=1}^{m-1} \frac{M^{p} L^{k p}}{2^{p} n^{p}}[\varphi(n x, 0,-x)+\varphi(-n x, 0, x)]^{p}
\end{aligned}
$$

for all nonnegative integers $m$ and $l$ with $m>l \geq 0$ and $x \in G$,where $g(x)=\frac{f(x)-f(-x)}{2}$. Since the sequence $\left\{\frac{g\left(n^{m} x\right.}{n^{m}}\right\}$ is Cauchy for all $x \in G$, we can define a function $h: G \rightarrow Y$ by

$$
h(x)=\lim _{m \rightarrow \infty} \frac{g\left(n^{m} x\right)}{n^{m}}=\lim _{m \rightarrow \infty} \frac{f\left(n^{m} x\right)-f\left(-n^{m} x\right)}{2 n^{m}}, \quad x \in G .
$$

Moreover, letting $l=0$ and $m \rightarrow \infty$ in the last inequality yields

$$
\left\|\frac{f(x)-f(-x)}{2}-h(x)\right\| \leq \frac{M}{2 n \sqrt[p]{1-L^{p}}}[\varphi(n x, 0,-x)+\varphi(-n x, 0, x)]
$$

for all $x \in$ G. It follows from (3) and (13) that

$$
\|f(x)-h(x)\| \leq \frac{M^{2}}{2 n \sqrt[p]{1-L^{p}}}[\varphi(n x, 0,-x)+\varphi(-n x, 0, x)]+\frac{M}{2} \varphi(x,-x, 0)
$$

for all $x L G$.

The remaining proof is similar to the corresponding proof of Theorem 2.1. This completes the proof.

Remark 3.2. Let $\phi: G^{3} \rightarrow \mathbf{R}^{+}$and $f: G \rightarrow Y$ satisfy the assumptions of Theorem 3.1. Then, there exists a unique additive mapping $h: G \rightarrow Y$, defined by $h(x)=\lim _{k \rightarrow \infty} \frac{f\left(n^{k} x\right)}{n^{k}}$, such that

$$
\|f(x)-h(x)\| \leq \frac{M}{n \sqrt[p]{1-L^{p}}}[\varphi(n x, 0,-x)+n \varphi(x,-x, 0)]
$$

for all $x \in G$ using the similar argument to Theorem 3.1.

In particular, if a mapping $f: X \rightarrow Y$ with $f(0)=0$ satisfies the following functional inequality: 


$$
\|f(x)+f(y)+n f(z)\| \leq\left\|n f\left(\frac{x+y}{n}+z\right)\right\|+\theta_{1}\left(\|x\|^{r}+\|y\|^{r}+\|z\|^{r}\right)+\theta_{2}
$$

for all $x, y, z$ in a normed space $X$, where $0<r<1, \theta_{1}, \theta_{2}>0$, then there exists a unique additive mapping $h: X \rightarrow Y$ such that

$$
\|f(x)-h(x)\| \leq \frac{M}{\sqrt[p]{n^{p}-n^{p r}}}\left(\left(n^{r}+2 n+1\right) \theta_{1}\|x\|^{r}+(n+1) \theta_{2}\right)
$$

for all $x \in X$, by considering $L:=n^{r-1}$.

Theorem 3.3. Suppose that a mapping $f: G \rightarrow Y$ satisfies the functional inequality

$$
\|f(x)+f(y)+n f(z)\| \leq\left\|n f\left(\frac{x+y}{n}+z\right)\right\|+\varphi(x, y, z)
$$

for all $x, y, z \in \mathrm{G}$ and there exists a constant $L$ with $0<L<1$ for which the perturbing function $\phi: G^{3} \rightarrow \mathbf{R}^{+}$satisfies

$$
\varphi\left(\frac{x}{n}, \frac{y}{n}, \frac{z}{n}\right) \leq \frac{L}{n} \varphi(x, y, z)
$$

for all $x, y, z \in G$. Then, there exists a unique additive mapping $h: G \rightarrow Y$, defined as $h(x) \lim _{k \rightarrow \infty} \frac{n^{k}}{2}\left(f\left(\frac{x}{n^{k}}\right)-f\left(-\frac{x}{n^{k}}\right)\right)$, such that

$$
\|f(x)-h(x)\| \leq \frac{M^{2} L}{2 n \sqrt[p]{1-L^{p}}}[\varphi(n x, 0,-x)+\varphi(-n x, 0, x)]+\frac{M}{2} \varphi(x,-x, 0)
$$

for all $x \in G$.

Proof. We observe that $f(0)=0$ because $\phi(0,0,0)=0$, which follows from the condition $\varphi(0,0,0) \leq \frac{L}{n} \varphi(0,0,0)$. It follows from the inequality (11) and (14) that

$$
\begin{aligned}
\left\|g(x)-n^{m} g\left(\frac{x}{n^{m}}\right)\right\|^{p} & \leq \frac{M^{p}}{2^{p}} \sum_{i=0}^{m-1} n^{i p}\left[\varphi\left(\frac{x}{n^{i}}, 0,-\frac{x}{n^{i+1}}\right)+\varphi\left(-\frac{x}{n^{i}}, 0, \frac{x}{n^{i+1}}\right)\right]^{p} \\
& \leq \frac{M^{p}}{2^{p} n^{p}} \sum_{i=0}^{m-1} L^{(i+1) p}[\varphi(n x, 0,-x)+\varphi(-n x, 0, x)]^{p}
\end{aligned}
$$

for all $x \in G$, where $g(x)=\frac{f(x)-f(-x)}{2}, x \in G$.

The remaining proof is similar to the corresponding proof of Theorem 2.1. This completes the proof.

Remark 3.4. Let $\phi: G^{3} \rightarrow \mathbf{R}^{+}$and $f: G \rightarrow Y$ satisfy the assumptions of Theorem 3.3. Then, there exists a unique additive mapping $h: G \rightarrow Y$, defined by $h(x)=\lim _{k \rightarrow \infty} n^{k} f\left(\frac{x}{n^{k}}\right)$, such that

$$
\|f(x)-h(x)\| \leq \frac{M L}{n \sqrt[p]{1-L^{p}}}[\varphi(n x, 0,-x)+n \varphi(x,-x, 0)]
$$

for all $x \in G$ using the similar argument to Theorem 3.3.

In particular, if a mapping $f: X \rightarrow Y$ satisfies the following functional inequality:

$$
\|f(x)+f(y)+n f(z)\| \leq\left\|n f\left(\frac{x+y}{n}+z\right)\right\|+\theta_{1}\left(\|x\|^{r}+\|y\|^{r}+\|z\|^{r}\right)
$$


for all $x, y, z$ in a normed space $X$, where $r>1, \theta_{1}>0$, then there exists a unique additive mapping $h: X \rightarrow Y$ such that

$$
\|f(x)-h(x)\| \leq \frac{M}{\sqrt[p]{n^{p r}-n^{p}}}\left(n^{r}+2 n+1\right) \theta_{1}\|x\|^{r}
$$

for all $x \in X$, by considering $L:=n^{1-r}$.

\section{Acknowledgements}

The authors would like to thank the referees and the editors for carefully reading this article and for their valuable comments. This study was supported by the Basic Research Program through the National Research Foundation of Korea funded by the Ministry of Education, Science and Technology (No. 2011-0002614).

\section{Authors' contributions}

All authors carried out the proof. All authors conceived of the study, and participated in its design and coordination All authors read and approved the final manuscript.

\section{Competing interests}

The authors declare that they have no competing interests.

Received: 2 April 2011 Accepted: 31 October 2011 Published: 31 October 2011

\section{References}

1. Ulam, SM: A Collection of the Mathematical Problems. Interscience Publ. New York (1960)

2. Hyers, DH: On the stability of the linear functional equation. Proc Natl Acad Sci USA. 27, 222-224 (1941). doi:10.1073/ pnas.27.4.222

3. Aoki, T: On the stability of the linear transformation in Banach spaces. J Math Soc Japan. 2, $64-66$ (1950). doi:10.2969/ jmsj/00210064

4. Bourgin, DG: Classes of transformations and bordering transformations. Bull Am Math Soc. 57, 223-237 (1951). doi:10.1090/S0002-9904-1951-09511-7

5. Rassias, TM: On the stability of the linear mapping in Banach spaces. Proc Am Math Soc. 72, 297-300 (1978). doi:10.1090/S0002-9939-1978-0507327-1

6. Găvruta, P: A generalization of the Hyers-Ulam-Rassias stability of approximately additive mappings. J Math Anal Appl. 184, 431-436 (1994). doi:10.1006/imaa.1994.1211

7. Jung, S: On the Hyers-Ulam-Rassias stability of approximately additive mappings. J Math Anal Appl. 204, 221-226 (1996). doi:10.1006/jmaa.1996.0433

8. Rassias, TM: The stability of mappings and related topics, in 'Report on the 27th ISFE'. Aequ Math. 39, 292-293 (1990)

9. Gajda, Z: On the stability of additive mappings. Int J Math Math Sci. 14, 431-434 (1991). doi:10.1155/ S016117129100056X

10. Rassias, TM, Semrl, P: On the behaviour of mappings which do not satisfy Hyers-Ulam-Rassias stability. Proc Am Math Soc. 114, 989-993 (1992). doi:10.1090/S0002-9939-1992-1059634-1

11. Rassias, JM: On approximation of approximately linear mappings by linear mappings. J Funct Anal. 46, 126-130 (1982). doi:10.1016/0022-1236(82)90048-9

12. Rassias, JM: On approximation of approximately linear mappings by linear mappings. Bull Sci Math. 108, 445-446 (1984)

13. Gao, ZX, Cao, HX, Zheng, WT, Xu, L: Generalized Hyers-Ulam-Rassias stability of functional inequalities and functional equations. J Math Inequal. 3(1), 63-77 (2009)

14. Moghimi, M, Najati, A, Park, C: A functional inequality in restricted domains of Banach modules. Adv Difference Equations 14 (2009). 2009, Article ID 973709

15. Moslehian, MS, Najati, A: An application of a fixed point theorem to a functional inequality. Fixed Point Theory. 10(1), 141-149 (2009)

16. Najati, A, Zamani, EG: Stability of a mixed additive and cubic functional equation in quasi-Banach spaces. J Math Anal Appl. 342, 1318-1331 (2008). doi:10.1016/j.jmaa.2007.12.039

17. Najati, A, Moradlou, F: Stability of a quadratic functional equation in quasi Banach spaces. Bull Korean Math Soc. 45 , 587-600 (2008)

18. Najati, A, Lee, J, Park, C: On a Cauchy-Jensen functional inequality. Bull Malaysian Math Sci Soc. 33(2):253-263 (2010)

19. Benyamini, Y, Lindenstrauss, J: Geometric Nonlinear Functional Analysis. American Mathematical Society, Providence1 (2000) Colloq. Publ. vol. 48

20. Rolewicz, S: Metric Linear Spaces. PWN-Polish Scientific Publishers, Reidel, Warszawa, Dordrecht (1984)

21. Najati, A, Moghimi, MB: Stability of a functional equation deriving from quadratic and additive function in quasi-Banach spaces. J Math Anal Appl. 337, 399-415 (2008). doi:10.1016/j.jmaa.2007.03.104

doi:10.1186/1029-242X-2011-102

Cite this article as: Kim and Son: Approximate Cauchy functional inequality in quasi-Banach spaces. Journal of Inequalities and Applications 2011 2011:102. 